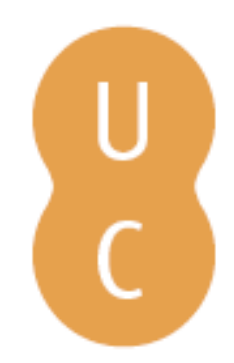

\title{
pommalina
}

\section{Efeito da ambiguidade temática no cálculo de métricas de paisagem}

Autor(es): $\quad$ Santos, Jorge Filipe dos

Publicado por: Imprensa da Universidade de Coimbra

URL

persistente:

URI:http://hdl.handle.net/10316.2/37065

DOI:

DOI:http://dx.doi.org/10.14195/978-989-26-0983-6_12

Accessed : $\quad$ 26-Apr-2023 12:17:16

A navegação consulta e descarregamento dos títulos inseridos nas Bibliotecas Digitais UC Digitalis, UC Pombalina e UC Impactum, pressupõem a aceitação plena e sem reservas dos Termos e Condições de Uso destas Bibliotecas Digitais, disponíveis em https://digitalis.uc.pt/pt-pt/termos.

Conforme exposto nos referidos Termos e Condições de Uso, o descarregamento de títulos de acesso restrito requer uma licença válida de autorização devendo o utilizador aceder ao(s) documento(s) a partir de um endereço de IP da instituição detentora da supramencionada licença.

Ao utilizador é apenas permitido o descarregamento para uso pessoal, pelo que o emprego do(s) título(s) descarregado(s) para outro fim, designadamente comercial, carece de autorização do respetivo autor ou editor da obra.

Na medida em que todas as obras da UC Digitalis se encontram protegidas pelo Código do Direito de Autor e Direitos Conexos e demais legislação aplicável, toda a cópia, parcial ou total, deste documento, nos casos em que é legalmente admitida, deverá conter ou fazer-se acompanhar por este aviso. 


\section{$\forall$ \\ TAS DAS I JORNADAS LUSÓFONAS DE CIÊNCIAS E TECNOLOGIAS DE INFORMAÇÃO GEOGRÁFICA}

Editores

José Gomes dos Santos

Cidália Fonte

Rui Ferreira de Figueiredo

Alberto Cardoso

Gil Gonçalves

José Paulo Almeida

Sara Baptista 


\section{A RTIGO 12 \\ EFEITO DA AMBIGUIDADE TEMÁTICA NO \\ CÁlCulo de Métricas de PAisAgem}

SANTOS, Jorge Filipe dos

Instituto Tecnológico Vale Desenvolvimento Sustentável

Rua Boaventura da Silva, 955. Nazaré. 66055-090. Belém/PA - Brasil

Tel: +55 (91) 32135543 ; email: jorge.filipe@itv.org

\section{RESUMO}

Este estudo pretende avaliar a influência da ambiguidade da classificação por objetos do sistema eCognition sobre o cálculo de métricas de paisagem. As métricas espaciais ou de paisagem são indicadores quantitativos obtidos a partir de cartas temáticas e permitem caracterizar a relação entre padrões espaciais e processos ecológicos. A execução de cartas temáticas é um processo que incorpora e gera incerteza. Consequentemente, é natural que seja dada importância à propagação dessa incerteza temática aos produtos derivados dessas cartas, como é o caso das métricas espaciais. Neste trabalho, o estudo estará focado na ambiguidade da classificação de imagens de satélite, sendo ignoradas outras fontes de incerteza temática. A ambiguidade temática será modelada através de um processo de simulação estocástica, onde serão geradas várias realizações da classificação de uma mesma imagem. O caso prático que será apresentado insere-se no contexto do projeto URBIS Amazônia, que tem como objetivo o estudo dos processos de urbanização da Amazónia brasileira. Espera-se perceber com este estudo qual pode ser o impacto da ambiguidade temática produzida pelo sistema Trimble eCognition Developer ${ }^{\mathrm{TM}}$, durante a classificação de uma imagem dos satélites Rapid Eye, sobre os valores das métricas espaciais obtidas com base nessa classificação. 


\title{
PALAVRAS-CHAVE
}

Ambiguidade, Carta temática, Métricas espaciais, Simulação estocástica.

\section{EFFECT OF THEMATIC AMBIGUITY ON}

\section{LANDSCAPE METRICS CALCULATION}

\begin{abstract}
This study aims to assess the influence of the classification ambiguity generated by object-based image analysis software (eCognition) on the landscape metrics calculation. Spatial or landscape metrics are quantitative indicators obtained from thematic maps that allow to characterize the relationship between spatial patterns and ecological processes. The production of thematic maps is a process that incorporates and generates uncertainty. Therefore, it is important to study the effect of this uncertainty propagation to the derivative products of these maps like the spatial metrics. In this work, the study will focus on the ambiguity of satellite images classification. Other sources of thematic uncertainty will be ignored. The thematic ambiguity will be modeled through a process of stochastic simulation, where several outputs of the classification will be generated for the same image. The case study to be presented here is developed under the URBIS Amazonia project framework, which aims to study the processes of urbanization in the Brazilian Amazon. It is expected to understand from this study how much can be the impact of the thematic ambiguity produced by Trimble eCognition Developer ${ }^{\mathrm{TM}}$ system during the classification process on the spatial metrics values derived from a Rapid Eye satellite image classification.
\end{abstract}

\section{KEYWORDS}

Ambiguity, Thematic map, Spatial metrics, Stochastic simulation.

\section{INTRODUÇÃO}

As métricas espaciais foram desenvolvidas no âmbito da área da ecologia da paisagem (landscape ecology) cuja terminologia foi criada por Carl Troll em 1939 (TURNER, 1989; TURNER, 2005; METZGER, 2008). A moderna Ecologia da Paisagem estuda as variações da paisagem em múltiplas escalas espaciais e temporais (BOTEQUILHA LEITÃO \& AHERN, 2002; WU, 2004; CUSHMAN et al., 2008; STEINIGER \& HAY, 2009; WU, 2011). A capacidade de descrever quantitativamente a estrutura da paisagem é uma condição necessária para o estudo das funções e das mudanças 
da paisagem. Para este fim, várias dezenas de métricas têm surgido na ecologia da paisagem (CUSHMAN et al., 2008; MCGARICAL et al., 2012). Todas estas métricas são definidas direta ou indiretamente com base no conceito de patch. Neste contexto, costuma traduzir-se patch por fragmento ou elemento de um mosaico da paisagem (MCGARICAL et al.., 2012). Do ponto de vista das ciências da informação geográfica, esses fragmentos podem ser considerados de forma simplificada como os polígonos de uma carta temática que delimitam as diferentes classes.

As cartas utilizadas neste contexto são produzidas normalmente através de dados e de metodologias de sensoriamento remoto (HEROLD et al., 2002). As incontornáveis simplicações e imperfeições que caracterizam este processo dão origem a uma informação com um determinado grau de incerteza que afecta o cálculo das métricas (SHAO \& WU, 2008). A incerteza temática tem vindo a ser estudada ao longo das últimas décadas, tendo sido apontadas algumas metodologias para tratar esta questão (ver, por exemplo, CONGALTON, 1991; WOODCOCK \& GOPAL, 2000; STEHMAN, 2001 ou FOODY, 2002). A incerteza das métricas de paisagem já foram analisadas segundo várias perspectivas por alguns autores como ARNOT (2004, 2007), DENDONCKER et al. (2008), LECHNER et al. (2012) e SHAO \& WU (2008). Neste caso, o problema da incerteza temática não será abordado na sua globalidade, mas apenas do ponto de vista dos resultados obtidos por uma classificação produzida pelo sistema eCognition. Este software está especialmente vocacionado para a classificação de imagens orientada por objetos (BAATZ \& SCHÄPE, 2000). Os objetos da imagem são polígonos resultantes de uma segmentação da imagem, no sentido de agrupar pixels homogéneos. O processo de classificação supervisada produz para cada objeto um grau de pertença relativo a cada uma das classes definidas a priori e para as quais foram indicadas amostras. Cada objeto é classificado de acordo com a pertença máxima. No entanto, normalmente, um grupo significativo desses objetos apresenta graus de pertença a outras classes muito próximos do máximo, gerando assim ambiguidade (ANTUNES \& LINGNAU, 2005). Este facto é fonte de incerteza, dado que os graus de pertença obtidos são resultado de um processo não isento de limitações a vários níveis, desde o processo 
captação da imagem, até à recolha das amostras na imagem e mesmo devido à natureza das classes utilizadas (FOODY, 2002; BENZ et al., 2004, 243). Irá então ser considerado que o processo de classificação tem uma componente aleatória induzida pelos polígonos de classe ambígua. Para avaliar o impacto desse fenómeno no cálculo das métricas espaciais, serão geradas várias realizações da classificação em que os polígonos ambíguos são classificados com base numa probabilidade uniforme, proporcional aos maiores graus de pertença que lhes foram atribuídos. A avaliação estatística dos valores das métricas obtidos a partir das realizações da classificação permitirá quantificar o impacto desta ambiguidade.

\section{APRESENTAÇÃo DOS DADOS}

As métricas espaciais podem ser uma ferramenta importante no estudo da evolução das cidades amazónicas (Ramos, 2014,33-37). Nesse sentido, foram obtidas imagens Rapid $\mathrm{Eye}^{\mathrm{TM}}$ para algumas dessas cidades. Para gerar as cartas temáticas a partir dessas imagens, estão a ser avaliadas várias alternativas, entre as quais se encontra o uso do sistema eCognition. Neste caso, foi utilizada uma imagem Rapid Eye de 2011 que cobre a cidade de Santarém do Pará e seu entorno (ver Figura 1).

As imagens Rapid Eye são compostas por cinco bandas espectrais, sendo três bandas do visível (RGB), uma banda do Infravermelho próximo (NIR) e outra no Red Edge (RE), que se situa entre as bandas R e NIR. A resolução espacial original de cada banda é de 6,5 metros, mas após a ortorretificação as bandas são reamostradas para uma resolução de 5 metros. A resolução radiométrica é de 12 bits. O produto usado tem um nível de processamento 3A, tendo correções relativas ao sensor, à radiometria e à geometria, incluindo ortorretificação. O sistema cartográfico é o WGS84/UTM, zona 21S. Neste caso foi cortada uma faixa no sul de imagem, por se encontrar coberta por bastantes nuvens e por ser uma zona afastada da cidade, que é foco do estudo. 


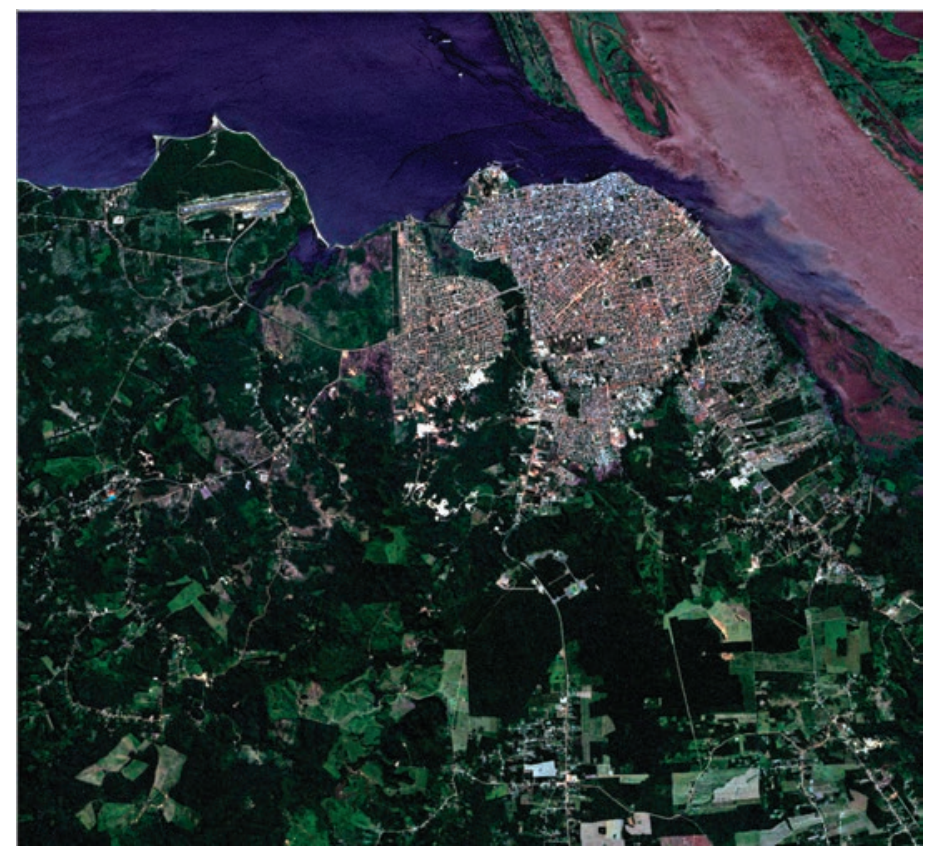

Figura 1 - Composição colorida usando as bandas do visível (RGB) da imagem Rapid Eye 2137925_2011-07-30_RE3_3A_166642 utilizada no estudo (com realce de brilho em editor de imagem)

\section{METOdOLOGiA}

A escolha das classes foi orientada segundo as necessidade do projecto, que pretende estudar essencialmente a ocupação urbana. Selecionaram-se assim as classes ÁGUA, SOLO, FLORESTA, OUTRA VEGETAÇÃO e ÁREAS IMPERMEABILIZADAS. A base metodológica para a produção do mapa temático a partir da imagem Rapid Eye fundamentou-se no uso de uma classificação supervisada orientada por objetos. O primeiro passo de uma classificação desse tipo é o agrupamento de pixels com um certo grau de semelhança, através de um processo de segmentação da imagem. Neste trabalho, foi testada a segmentação multi-resolução com vários valores de parâmetros que o programa eCognition permite (Baatz \& Schäpe, 2000), no sentido de obter um bom equilíbrio entre o detalhe e a quantidade total de polígonos a classificar. Os parâmetros escolhidos foram os seguintes: Scale Parameter $=100$, Shape $=0.1$ e Compactness $=0.5$. Utilizaram-se todas as 5 bandas da imagem neste processo, às quais foram dados pe- 
sos iguais. Para fazer a classificação supervisada dos polígonos (objetos) obtidos foram escolhidas amostras sobre a imagem por forma a serem representativas de cada uma das classes. Foi efetuada uma análise das variáveis que melhor poderiam separar as classes, com base nas amostras indicadas, usando o método de Optimização do Espaço de Variáveis (Feature Optimization Space) do eCognition. Este método é baseado na norma euclidiana calculada entre as amostras de todas as classes. A melhor separação de classes é considerada como aquela que apresenta a maior das distâncias mínimas entre as classes menos separáveis (Leduc, 2004, Laliberte, 2012). Deste processo resultou a matriz de separação de classes para as seis variáveis selecionadas, que pode ser observada na Tabela 1. Pode observar-se nessa tabela que a maior dificuldade estará em separar o solo exposto das superfícies impermeabilizadas, pois são as classes que apresentam menor distância de separação.

Tabela 1 - Matriz de separação de classes

\begin{tabular}{|c|c|c|c|c|c|}
\hline & FLORESTA & $\begin{array}{c}\text { OUTRA } \\
\text { VEGETAÇÃO }\end{array}$ & SOLO & $\begin{array}{c}\text { ÁREAS } \\
\text { IMPERMEABILIZADAS }\end{array}$ & ÁGUA \\
\hline FLORESTA & 0 & 11.03 & 22.38 & 29.68 & 7.69 \\
\hline $\begin{array}{c}\text { OUTRA } \\
\text { VEGETAÇÃO }\end{array}$ & & 0 & 7.23 & 18.30 & 17.78 \\
\hline SOLO & & & 0 & 2.87 & 38.75 \\
\hline $\begin{array}{c}\text { ÁREAS } \\
\text { IMPERMEABILIZADAS }\end{array}$ & & & & & 45.91 \\
\hline ÁGUA & & & & & 0 \\
\hline
\end{tabular}

As variáveis selecionadas foram as seguintes: Stddev of area represented by segments, quantile[50](B), Stddev B, Mean B, Mean RE e Stddev RE. Seguidamente foi aplicado o método de classificação Nearest Neighbor baseada em lógica difusa que é oferecido por esse mesmo programa (Baatz \& Schäpe, 1999). Como se pode ver pelas variáveis escolhidas, esta classificação permite o uso de outros valores para além da radiometria direta da imagem.

Como resultado desta classificação, é obtida uma matriz que contém o grau de pertença de cada polígono a cada uma das classes consideradas. 
Por defeito, a classe que é atribuída automaticamente pelo eCognition a cada polígono corresponde ao valor máximo de pertença. O valor de pertença do polígono a cada uma das classes pode variar, sem restrições, entre 0 e 1.

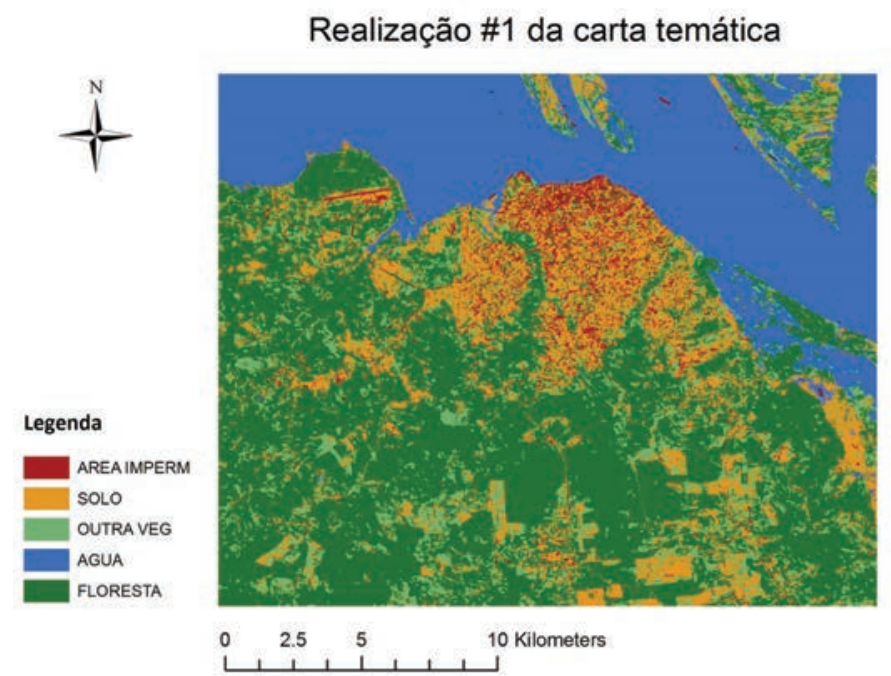

Figura 2 - Realização \#1 da carta temática obtida a partir da imagem da Figura 1

Para simular a ambiguidade escolheu-se a simulação estocástica como método de modelação deste tipo de incerteza. Achou-se que este método seria o mais adequado devido à natureza do problema e à facilidade de implementação através de uma rotina que foi construída em Matlab ${ }^{\mathrm{TM}}$ para esse efeito. Essa rotina permite, num primeiro passo, identificar os polígonos para os quais pode existir ambiguidade temática. Isso foi feito comparando as pertenças máximas de um polígono a uma classe com os outros graus de pertença do mesmo polígono às outras classes. Após alguns testes, foi considerado que uma proximidade de pertenças até $10 \%$ seria razoável, isto é, todos os polígonos que tenham classes cuja diferença de pertença em relação à máxima fosse inferior a 0.1 seriam considerados como ambíguos quanto à classe que lhe foi atribuída. Apenas os polígonos ambíguos, segundo a condição referida atrás, foram considerados nesta simulação. Numa segunda parte da rotina, considerou-se que 
a probabilidade de atribuir uma certa classe a cada um desses polígonos seria diretamente proporcional ao grau de pertença atribuído durante o processo de classificação gerado pelo algoritmo do eCognition. Esse princípio foi usado para escolher aleatoriamente as classes dos polígonos ambíguos. Foi assim gerado um número significativo de 30 realizações da carta temática para modelar a ambiguidade em causa.

Para calcular as métricas espaciais foi utilizado o programa Fragstats. Nesse programa, a métricas estão agrupadas em 3 níveis: por fragmentos, por classes e para a totalidade da paisagem (ver MCGARICAL et al., 2012). Iremos considerar a título de exemplo, neste trabalho, apenas algumas das métricas mais utilizadas por classes e para a totalidade da paisagem, que iremos enunciar de seguida:

Densidade de Polígonos (PD-Patch Density): quantidade de polígonos por área.

Densidade de Arestas (ED-Edge Density): razão entre o número de arestas que separam duas classes diferentes e a respetiva área.

Área Média dos Polígonos (AREA_MN-Mean Patch Area): área média dos polígonos.

Raio de Giro Médio (GYRATE_MN- Mean Radius of Gyration): média das distâncias das células de um fragmento ao seu centróide.

Índice de Diversidade de Shannon (SHDI-Shannon's diversity Index): $-\sum^{n}{ }_{i=1}\left(P_{i} \log P_{i}\right), P_{i}$ é a percentagem de área ocupada pela classe $i$. Tem como base a noção de entropia da teoria da informação.

Índice de Justaposição e de Intercalação (IJI-Interspersion and Juxtaposition Index): $-\sum^{n}{ }_{i=1} \sum_{k=i+1}^{n}($ EiklogEik $) / \log (m(m-1) / 2), E_{i k}$ é o comprimento da aresta entre a classe $i$ e a classe $k$ e $m$ o número de classes.

Para calcular estas métricas espaciais, foi gerado um ficheiro geotiff para cada uma das 30 realizações da carta temática obtidas pela simulação estocástica, através da rotina $M a t l a b^{\mathrm{TM}}$, referida anteriormente. Esses ficheiros foram usados como dados de entrada no programa Fragstats (MCGARICAL et al., 2012), onde foram calculadas as correspondentes 30 realizações de cada uma das métricas. Para conhecer alternativas de 
software para o cálculo de métricas, consultar STEINIGER \& HAY (2009).

No sentido de tornar mais clara a metodologia utilizada, foi elaborado um fluxograma que pode ser observado na Figura 3.
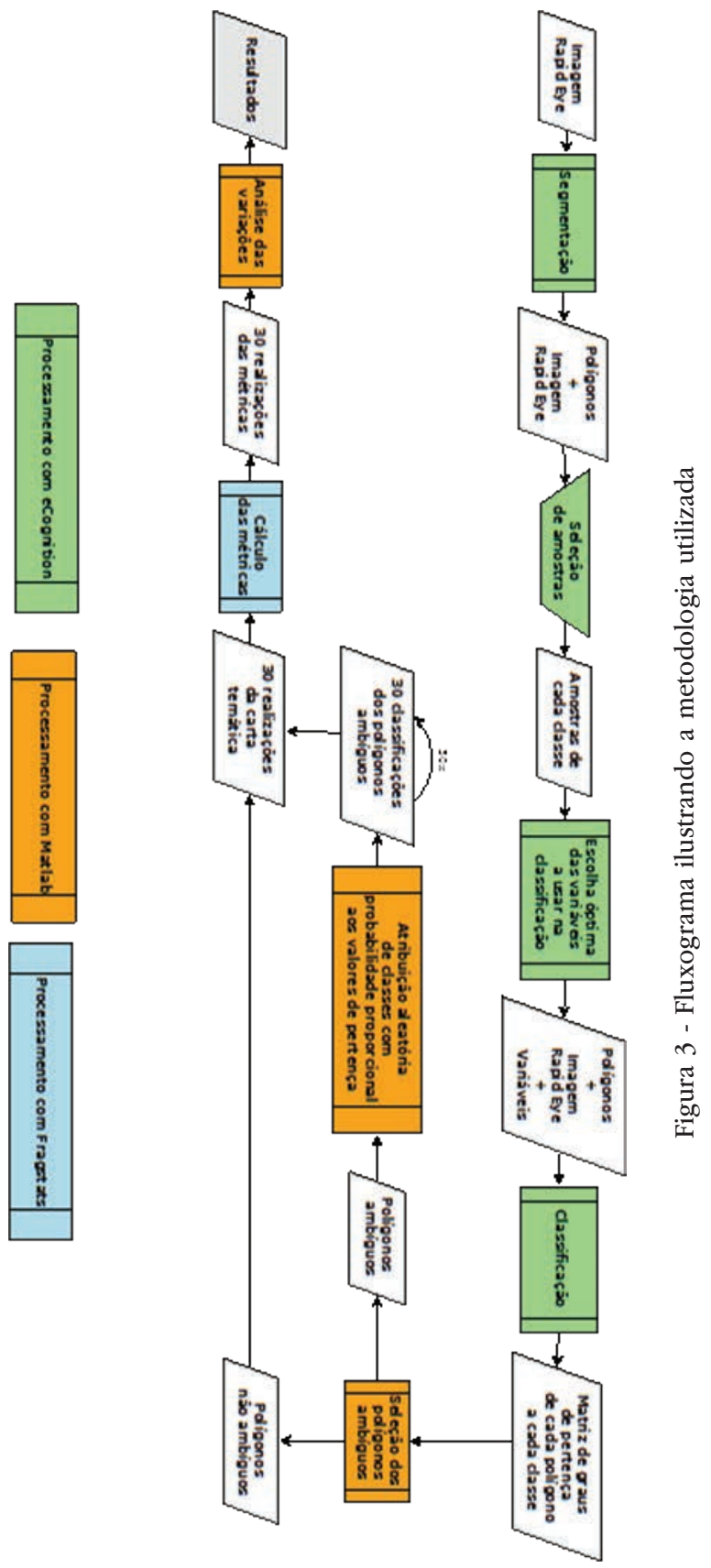


\section{DisCUSSÃo DOS RESULTADOS E CONCLUSÕES}

Os valores obtidos pelo processamento descrito atrás foram analisados, no sentido de perceber qual poderá ser a influência da ambiguidade da classificação sobre os valores das métricas dependentes dessa classificação.

Tabela 2: Desvios-padrão das 30 realizações das métricas, por classe, normalizados pelas respetivas médias (em percentagem)

\begin{tabular}{|c|c|c|c|c|c|}
\hline Classe $\backslash$ Métrica & PD & ED & AREA_MN & GYRATE_MN & IJI \\
\hline AREA_IMP & 1.2 & 1.0 & 1.9 & 0.9 & 1.3 \\
\hline SOLO & 1.1 & 0.5 & 1.4 & 1.0 & 0.2 \\
\hline OUTRA_VEG & 0.9 & 0.8 & 1.5 & 0.6 & 0.5 \\
\hline AGUA & 2.6 & 1.2 & 2.6 & 2.3 & 0.5 \\
\hline FLORESTA & 1.0 & 0.5 & 1.0 & 1.4 & 0.6 \\
\hline
\end{tabular}

Tabela 3: Desvios-padrão das 30 realizações das métricas, para toda a paisagem, normalizados pelas respetivas médias (em percentagem)

\begin{tabular}{|c|c|c|c|c|c|c|}
\hline Métrica & PD & ED & AREA_MN & GYRATE_MN & IJI & SDHI \\
\hline TOTAL & 0.4 & 0.4 & 0.4 & 0.3 & 0.2 & 0.1 \\
\hline
\end{tabular}

Com o intuito de quantificar a variação que ocorreu entre as 30 realizações de cada uma das métricas, foram calculados os desvios-padrão de cada uma delas. Os valores das métricas têm grandezas bastante diferentes entre si. Por isso, foi feita uma normalização dos desvios-padrão obtidos, dividindo cada um deles pela respectiva média e convertendo esses valores em percentagem. A Tabela 2 mostra os desvios-padrão normalizados das 30 realizações de cada métrica de paisagem considerada neste estudo. Supondo que essas realizações são representativas das variações que a classificação pode sofrer devido à ambiguidade da atribuição da classe a cada polígono pelo algoritmo do sistema eCognition, podemos observar que essas variações rondam o valor de $1 \%$. Podemos então concluir que a ambiguidade que pode afetar o processo de classificação executado pelo eCognition não tem influência signiticativa no cálculo das métricas de paisagem das classes em causa. Por maioria de razão, observando a Tabela 3, podemos concluir também que as métricas espaciais calculadas sobre toda a paisagem são ainda menos afetadas por essa incerteza.

A título de conclusão final, poderemos dizer que, nas condições da 
metodologia utilizada, a ambiguidade temática gerada pela classificação Nearest Neighbor, que está implementada no eCognition, não tem uma influência significativa nos valores obtidos para as métricas de paisagem.

Será futuramente feito um trabalho de recolha de amostras para as várias classes no sentido de verificar a exatidão temática da classificação obtida. Será assim possível efetuar um estudo mais completo sobre a influência da incerteza temática na determinação das métricas espaciais.

\section{AGRADECIMENTOS}

O autor agradece à Diretoria de Vendas da Rapid Eye para a América Latina pela cedência para fins de investigação da imagem Rapid Eye utilizada neste estudo.

\section{BIBLIOGRAFIA}

ANTUNES, Alzir. F. B., \& Lingnau, Christel (2005). Determinação da acurácia temática de dados oriundos da classificação digital de objetos por meio de lógica fuzzy. In J. C. N. Epiphanio, \& L. M. G. Fonseca (Eds.), XII Simpósio Brasileiro de Sensoriamento Remoto (pp. 3451-3459).

ARNOT, Charles \& Fisher, P. (2007). Mapping the ecotone with fuzzy sets. In Geographic uncertainty in environmental security (pp. 19-32). Springer Netherlands.

ARNOT, Charles (2004). Characterising uncertain landscape structure. PhD thesis, University of Leicester.

ARNOT, Charles et al. (2004). Landscape metrics with ecotones: pattern under uncertainty. Landscape Ecology, 19(2), 181-195.

BAATZ, Martin \& Schäpe, Arno (1999). Objected oriented and multiscale image analysis in semantic network. In 2nd International Symposium: Operationalization of Remote Sensing, 16-20 August, ITC, NL.

BAATZ, Martin \& Schäpe, Arno (2000). "Multiresolution segmentation-an optimization approach for high quality multi-scale image segmentation". In Angewandte geographische informationsverarbeitung, Edited by: Strobl, J., Blaschke, T. and Griesebner, G. 12-23. Heidelberg: Wichmann-Verlag.

BENZ, Ursula C. et al. (2004). Multi-resolution, object-oriented fuzzy analysis of remote sensing data for GIS-ready information. ISPRS Journal of photogrammetry and remote sensing, 58(3), 239-258. 
BOTEQUILHA LEITÃO, André, \& Ahern, Jack (2002). Applying landscape ecological concepts and metrics in sustainable landscape planning. Landscape and urban planning, 59(2), 65-93.

CONGALTON, Russell. G. (1991). A review of assessing the accuracy of classifications of remotely sensed data. Remote sensing of environment, 37(1), 35-46.

CUSHMAN, Samuel A. et al.(2008). Parsimony in landscape metrics: strength, universality, and consistency. Ecological indicators, 8(5), 691-703.

DENDONCKER, Nicolas et al. (2008). Exploring spatial data uncertainties in land-use change scenarios, International Journal of Geographical Information Science, 22:9, 1013-1030.

FOODY, Giles M. (2002). Status of land cover classification accuracy assessment. Remote sensing of environment, 80(1), 185-201.

HEROLD, Martin et al. (2002). The use of remote sensing and landscape metrics to describe structures and changes in urban land uses. Environment and Planning A, 34(8), 1443-1458.

LALIBERTE, Andrea S. et al. (2012). A comparison of three feature selection methods for object-based classification of sub-decimeter resolution UltraCam-L imagery. International Journal of Applied Earth Observation and Geoinformation, v. 15, p. 70-78.

LECHNER, Alex M. et al. (2012). Are landscape ecologists addressing uncertainty in their remote sensing data?. Landscape ecology, 27(9), 1249-1261.

LEDUC, François (2004). Feature space optimization prior to fuzzy image classification. In Proc. of the 7 th International Conference on Information Fusion (pp. 547-554).

MCGARIGAL, Kevin et al. (2012). FRAGSTATS v4: Spatial Pattern Analysis Program for Categorical and Continuous Maps. Computer software program produced by the authors at the University of Massachusetts, Amherst. Available at the following web site: http://www.umass.edu/landeco/research/fragstats/ fragstats.html

METZGER, Jean P. (2008). Landscape ecology: perspectives based on the 2007 IALE world congress. Landscape Ecology, 23(5), 501-504.

RAMOS, Frederico R. (2014). Três ensaios sobre a estrutura espacial urbana em cidades do Brasil contemporâneo: economia urbana e geoinformação na construção de novos olhares. Tese de doutorado, Fundação Getúlio Vargas, $177 \mathrm{p}$.

SHAO, Guofan \& Wu, Jianguo (2008). On the accuracy of landscape pattern analysis using remote sensing data. Landscape Ecology, 23(5), 505-511.

STEHMAN, Stephen V. (2001). Statistical rigor and practical utility in thematic map accuracy assessment. Photogrammetric Engineering and Remote Sensing, 
67(6), 727-734.

STEINIGER, Stefan \& Hay, Geoffrey J. (2009). Free and open source geographic information tools for landscape ecology. Ecological Informatics, 4(4), 183-195.

TURNER, Monica G. (1989). Landscape ecology: The effect of pattern on process. Annual review of ecology and systematics, 171-197.

TURNER, Monica G. (2005). Landscape ecology: what is the state of the science?. Annual Review of Ecology, Evolution, and Systematics, 319-344.

WOODCOCK, Curtis E., \& Gopal, Sucharita (2000). Fuzzy set theory and thematic maps: accuracy assessment and area estimation. International Journal of Geographical Information Science, 14(2), 153-172.

WU, Jianguo (2004). Effects of changing scale on landscape pattern analysis: scaling relations. Landscape Ecology, 19(2), 125-138.

WU, Jianguo et al. (2011). Quantifying spatiotemporal patterns of urbanization: The case of the two fastest growing metropolitan regions in the United States. Ecological Complexity, 8(1), 1-8. 
Série Documentos

Imprensa da Universidade de Coimbra

Coimbra University Press

2015

- U M

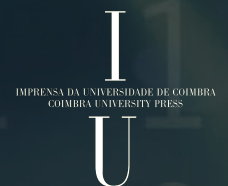

